\title{
Two-photon double ionization of the helium atom by ultrashort pulses
}

\author{
A. Palacios \\ Departamento de Química, Universidad Autónoma de Madrid, Madrid, Spain \\ D. A. Horner \\ Los Alamos National Laboratory, Theoretical Division,Los Alamos, NM 87545
}

\section{T. N. Rescigno}

Lawrence Berkeley National Laboratory, Chemical Sciences and Ultrafast X-ray Science Laboratory, Berkeley, CA 94720, USA

\section{W. McCurdy}

Lawrence Berkeley National Laboratory, Chemical Sciences and Ultrafast X-ray Science Laboratory, Berkeley, CA 94720, USA

Departments of Applied Science and Chemistry, University of California, Davis,CA 95616, USA

\begin{abstract}
Two-photon double ionization of the helium atom was the subject of early experiments at FLASH and will be the subject of future benchmark measurements of the associated electron angular and energy distributions. As the photon energy of a single femtosecond pulse is raised from the threshold for two-photon double ionization at $39.5 \mathrm{eV}$ to beyond the sequential ionization threshold at $54.4 \mathrm{eV}$, the electron ejection dynamics change from the highly correlated motion associated with nonsequential absorption to the much less correlated sequential ionization process. The signatures of both processes have been predicted in accurate $a b$ initio calculations of the joint angular and energy distributions of the electrons, and those predictions contain some surprises. The dominant terms that contribute to sequential ionization make their presence apparent several eV below that threshold. In two-color pump probe experiments with short pulses whose central frequencies require that the sequential ionization process necessarily dominates, a two-electron interference pattern emerges that depends on the pulse delay and the spin state of the atom.
\end{abstract}




\section{Introduction}

Experiments using pulses of femtosecond or attosecond durations are opening the path to probing electron dynamics in atoms and molecules on its intrinsic time scale. That time scale ranges from a few hundred attoseconds or shorter (the classical period of the first Bohr orbit in hydrogen is about 150 attoseconds), to several femtoseconds, which is the time scale of charge migration in small organic molecule with a few tens of atoms (Remacle \& Levine 2006, Lunnemann et al. 2008). A six-cycle 500 attosecond pulse corresponds to an energy of $50 \mathrm{eV}$, so it is evident that pulses short enough to probe electron dynamics in the time domain, even those containing only a few cycles of the radiation field, will frequently be energetic enough to ionize or multiply ionize the target atom or molecule and will generally be in the extreme UV or shorter wavelength regions of the spectrum. Thus an understanding of the fundamental processes that occur at these energies will be essential to designing and interpreting these experiments.

At FLASH, pioneering experiments in this energy regime have been made possible by the fact that free-electron lasers (V. Ayvazyan, et al. 2006) can reach the intensities needed for acquiring robust signals in measurements of multiphoton ionization, and which ultimately will be needed for measurements in pump/probe experiments involving more than one photon at UV or higher energies. Of course, the promise of ultrafast experiments in this energy domain has been also underscored by a large number of recent measurements using high harmonic generation, some of which are described by (Krausz \& Ivanov 2009) and (Sekikawa et al. 2004) for example. However, the intensities that can be reached at FLASH currently provide a significant advantage for multiphoton measurements in the XUV.

Some of the first experiments on multiphoton ionization of atoms (Kurka et al. 2009, Rudenko et al. 2008, Sorokin et al. 2007, Hasegawa et al. 2005) have already revealed the difficulties in their interpretation that pose a challenge to theory, and more such experiments are underway at FLASH and elsewhere (Ullrich 2009). Theoretical calculations of benchmark accuracy on simple systems have now become necessary for the analysis and understanding of observations that are necessarily carried out under conditions where a number of ionization channels compete with the one that is the focus of the measurement. The simplest target atom for such measurements is the helium atom, and here we focus on its two-photon double ionization by photons of one or two colors. The complete and unambiguous interpretation of those experiments will form the foundation for the application of multiphoton ionization measurements using free-electron lasers on more complicated atomic and molecular targets.

Unsurprisingly, two-photon double ionization of helium has been a subject of intense theoretical interest in recent years (Palacios et al. 2007, Palacios et al. 2008, Palacios et al. 2009a, Palacios et al. 2009b, Horner et al. 2007, Horner, McCurdy \& Rescigno 2008, Horner, Rescigno \& McCurdy 2008, Feist et al. 2008, Nikolopoulos \& Lambropoulos 2007, Ivanov \& Kheifets 2007, Foumouo et al. 2006, Kheifets \& Ivanov 2006, Hu et al. 2005, Feng \& van der Hart 2003, Piraux et al. 2003, Nikolopoulos \& Lambropoulos 2001, Colgan \& Pindzola 2002, Proulx et al. 1994, Shakeshaft 2007, Foumouo, Antoine, Piraux, Malegat, Bachau \& Shakeshaft 2008, Foumouo, Antoine $\&$ Piraux 2008). These studies, only a sampling of which are cited here, have most frequently focused on the energy range (40 to $54.4 \mathrm{eV}$ ) where double ionization must proceed by nonsequential absorption of two photons. In that energy range electron correlation must necessarily play an important role in the ionization mechanism, 
because the two electrons must share the energy of two photons in order to escape. Recent theoretical investigations have also focussed on the transition to the dynamics dominated by sequential absorption above $54.4 \mathrm{eV}$, and on what might be learned by pump/probe experiments above that threshold. Although the system only contains two electrons, its correlated double ionization dynamics upon absorption of two photons are not easy to describe in accurate, predictive calculations, as is evidenced by the fact that for a number of years there was disagreement on even the simplest measurable quantity associated with that process, namely the total two-photon double ionization cross section. Some consensus has by now been reached at this point.

In this article we will first review one of the theoretical methods used to date in $a b$ initio calculations on two-photon double ionization. In a survey of recent results we will describe the integral cross sections and some of the behavior of the joint angular distributions of the ejected electrons which reveal effects of electron correlation in the initial and final states. However, those angular distributions have been predicted to change when sufficiently short pulses are used, and we will discuss that effect, in which changing the pulse duration apparently changes the electron ejection dynamics that are observed. In the energy region just below the threshold for sequential double ionization at $54.4 \mathrm{eV}$ we will describe marked changes in both total and single differential cross sections in which a partial signature of the dominant contributions to sequential ionization can be seen below the energetic threshold for that process. Finally we will describe some recent surprises predicted theoretically for XUV pump/probe experiments in which a novel kind of interference pattern appears in the joint distribution of energy shared by the two electrons.

\section{Theory}

2.1. The extraction of double ionization amplitudes from solutions of the time-dependent Schrödinger equation

For two-electron atoms interacting with an intense UV or XUV pulse it is feasible to solve the time-dependent Schrödinger equation (TDSE) numerically using a grid representation for the coordinates of each electron. For intensities where up to three or four photons are absorbed, these solutions can be found to almost arbitrary accuracy with current computers and modern numerical algorithms, except very close to ionization thresholds, where very large grids are required to describe Rydberg states and slow continuum electrons, or for high energies, where the number of partial waves and the densities of the underlying grids required become prohibitively large.

Thus, the central issue for these calculations is not the solution of the TDSE to produce the wave packet describing the motion of the electrons during and after the pulse, although that may require many processors of a large computer. Instead the central problem is the calculation of the double ionization amplitudes from the resulting wave packets for the ejection of electrons of specific energies. The double ionization channel is in general a minor one, with single ionization being much more probable. Moreover, double ionized states lie in the Coulomb three-body breakup continuum where the Coulomb interactions continue to couple the motions of the electrons over long distance. In this section we summarize the methods we use to solve this problem in a way that allows the practical extraction of the double ionization probabilities for energies within the bandwidth(s) of the pulse(s) used to create an ionizing wave packet, without necessitating the use of prohibitively large grids or 
explicit propagation to long times following the pulses.

Consider an atom initially in its ground state that is subjected to a radiation pulse that starts at $t=0$ and ends at $t=T$. We solve the TDSE using the dipole approximation for the laser-atom interaction,

$$
i \frac{\partial}{\partial t} \Psi(t)=\mathcal{H}(t) \Psi(t)
$$

where $\mathcal{H}(t)=H_{0}+V_{t}$, with $H_{0}$ being the atomic Hamiltonian and $V_{t}$ the laser-atom interaction. In the length gauge the interaction is given in terms of the electric field is $\mathbf{E}(t)$, and the dipole operator $\boldsymbol{\mu}=-e \sum_{i} \mathbf{r}_{i}$. The calculations we present here were performed primarily in the length gauge with velocity gauge calculations used in independent convergence tests. Our pulses have a sine-squared envelope and are of total duration $T$ so that for a a photon energy $\omega$, the electric field and interaction potential (for a single pulse, for example) can be written

$$
\begin{aligned}
& \mathbf{E}(t)=\left\{\begin{array}{cc}
E_{0} F_{\omega}(t) \hat{\boldsymbol{\epsilon}} & t \in[0, T] \\
0 & \text { elsewhere }
\end{array}\right. \\
& V_{t}=\mathbf{E}(t) \cdot \boldsymbol{\mu},
\end{aligned}
$$

where $E_{0}$ is the maximum field strength, and $\hat{\boldsymbol{\epsilon}}$ is the polarization vector. The time dependence of the field is given by the function pulse, $F_{\omega}(t)$,

$$
F_{\omega}(t)=\sin ^{2}\left(\frac{\pi}{T} t\right) \sin (\omega t) .
$$

The pulse has ended at $t=T$, but the outgoing electrons are still interacting with each other and the nucleus as the system continues to propagate under the influence of the field-free Hamiltonian $H_{0}$. Nonetheless, in principle the double ionization probability for electrons to be ejected with specific momenta are determined already at $t=T$ as we can see by expanding $\Psi(T)$ in terms of sums and integrals over solutions of the time-independent Schrödinger equation. If $\psi_{\text {bound }}\left(\mathbf{r}_{1}, \mathbf{r}_{2}\right)$ contains the contributions from the bound states of the atom, and $n$ labels bound states of $\mathrm{He}^{+}$, we can write

$$
\begin{aligned}
\Psi\left(\mathbf{r}_{1}, \mathbf{r}_{2}, T\right)= & \psi_{\text {bound }}\left(\mathbf{r}_{1}, \mathbf{r}_{2}\right)+\psi_{\text {single }}\left(\mathbf{r}_{1}, \mathbf{r}_{2}\right)+\psi_{\text {double }}\left(\mathbf{r}_{1}, \mathbf{r}_{2}\right) \\
= & \psi_{\text {bound }}\left(\mathbf{r}_{1}, \mathbf{r}_{2}\right)+\sum_{n} \int d k_{n}^{3} C\left(\mathbf{k}_{n}\right) \psi_{\mathbf{k}_{n}}^{-}\left(\mathbf{r}_{1}, \mathbf{r}_{2}\right) \\
& +\int d k_{1}^{3} \int d k_{2}^{3} C\left(\mathbf{k}_{1}, \mathbf{k}_{2}\right) \psi_{\mathbf{k}_{1}, \mathbf{k}_{2}}^{-}\left(\mathbf{r}_{1}, \mathbf{r}_{2}\right) .
\end{aligned}
$$

The coefficients $C\left(\mathbf{k}_{n}\right)$ and $C\left(\mathbf{k}_{1}, \mathbf{k}_{2}\right)$ of the singly ionized states, $\psi_{\mathbf{k}_{n}}^{-}\left(\mathbf{r}_{1}, \mathbf{r}_{2}\right)$ and doubly ionized states, $\psi_{\mathbf{k}_{1}, \mathbf{k}_{2}}^{-}\left(\mathbf{r}_{1}, \mathbf{r}_{2}\right)$, respectively, are the amplitudes for single and double ionization. However it would appear that to calculate them at $t=T$ we would either have to project on the exact singly and doubly ionized wave functions (which we do not know) or propagate to long enough times that the outgoing electrons are no longer interacting appreciably.

We have shown how to avoid the necessity for integrating to long times after the pulse, or for performing calculations with pulses of various lengths to determine rates of ionization proportional to $T$ and the Nth power of the intensity for Nphoton absorption. In the method we have developed (Palacios et al. 2007, Palacios et al. 2008, Palacios et al. 2009a), we construct a scattered wave which is the Fourier transform of the time-propagated the wave packet for a specific total energy $E$ :

$$
\Psi_{s c} \equiv-i \lim _{\gamma \rightarrow 0} \int_{0}^{\infty} d t e^{i\left(E+i \gamma-H_{0}\right) t} \Psi(T) .
$$


The function $\Psi_{s c}$ contains all the physical information about processes leading to final states at energy $E$ with its asymptotic behavior determined by the single and double ionization amplitudes. The solution of Eq. (6) is also a solution of the timeindependent equation

$$
\left(E-H_{0}\right) \Psi_{s c}=\Psi(T),
$$

with pure outgoing boundary conditions.

To solve Eq. (7) using a finite matrix representation of the atomic Hamiltonian, $H_{0}$, we need a way of applying those boundary conditions. However, they involve the complicated asymptotic form of the exact solution of the three-body Coulomb breakup problem (Alt \& Mukhamedzhanov 1993, Kadyrov et al. 2003, Mukhamedzhanov et al. 2006) which has proved extremely difficult to apply explicitly in numerical calculations. Escaping this difficulty by imposing pure outgoing wave boundary conditions implicitly is the purpose for which the method of exterior complex scaling (ECS) was developed. In the ECS method the electronic coordinates are scaled only beyond a radius $R_{0}$ by a complex phase factor according to $r \rightarrow R_{0}+\left(r-R_{0}\right) e^{i \eta}$, and the value of $R_{0}$ is chosen large enough that the wave packet can be assumed not to have reached that radius during the pulse. Thus our numerical grids must now be extended into the complex plane, but only beyond $R_{0}$. It is now understood (McCurdy et al. 2004), that solving Eq. (7), with the boundary condition that the solution vanish at the end of the complex portion of the numerical grid on which it is represented, is formally equivalent to applying pure outgoing boundary conditions, no matter how many electrons are being ejected.

It is worth mentioning that both the time propagation, for which we use the Crank-Nicolson method, and the solution of the driven equation (Eq. (7)), are performed with iterative linear equation solvers. For the time propagation, we use the fact that the wave packet varys little with each time step, so very few iterations are required for convergence. The solution of the final driven equation is more costly. In practice, the time spent in solving Eq. (7) is about the same as the time required to take a few hundred timesteps in the propagation.

The ionization amplitudes naturally appear in the asymptotic form of $\Psi_{s c}$ at a fixed energy. For an atomic target they can be extracted using surface integrals involving $\Psi_{s c}$ and atomic Coulomb functions (McCurdy et al. 2004). In particular, for double ionization, which is the focus of our attention here, we have

$$
\begin{aligned}
C\left(\mathbf{k}_{1}, \mathbf{k}_{2}\right)=\frac{1}{2} e^{i \chi} \int\left[\phi_{\mathbf{k}_{1}}^{-*}\left(\mathbf{r}_{1}\right) \phi_{\mathbf{k}_{\mathbf{2}}}^{-*} \nabla \Psi_{\mathrm{sc}}\left(\mathbf{r}_{1}, \mathbf{r}_{2}\right)\right. & \\
& \left.-\Psi_{\mathrm{sc}}\left(\mathbf{r}_{1}, \mathbf{r}_{2}\right) \nabla\left(\phi_{\mathbf{k}_{1}}^{-*}\left(\mathbf{r}_{1}\right) \phi_{\mathbf{k}_{\mathbf{2}}}^{-*}\left(\mathbf{r}_{2}\right)\right)\right] \cdot d \mathbf{S},
\end{aligned}
$$

where the two-electron gradient is $\nabla=\left(\nabla_{1}, \nabla_{2}\right)$, and the testing functions $\phi_{\mathbf{k}}^{-}$are momentum-normalized Coulomb functions with a nuclear $Z=2$. We emphasize that these are not approximations to the final state of the system, but instead are the appropriate functions to extract the asymptotic amplitudes from $\Psi_{s c}$ in the limit of a large volume enclosed by the surface integral, as can be shown from stationary phase arguments. In the formula for the double ionization amplitudes, $\chi$ is an irrelevant volume-dependent overall phase that makes no contribution to any physical observable.

The surface integral extraction of the amplitudes is performed on the real part of the grid, close to the ECS turning point. Several factors must be considered in deciding how large a grid is required. For very short pulses, the wave packet does not spread very far and the grid size is then determined by the asymptotic behavior of the 
scattered wave. For pulses on the order of a few fs or more, the grid size is determined by the minimum value of $R_{0}$ needed to contain the spreading wave packet and avoid unphysical reflections from the grid boundaries during the time propagation. For the results presented here, a value of $R_{0}=130$ bohr was found to be sufficient.

Now with the amplitudes $C\left(\mathbf{k}_{1}, \mathbf{k}_{2}\right)$ in hand we turn to the task of defining the double ionization cross sections in terms of them.

\subsection{Two-photon ionization cross sections}

To define the generalized cross section for the ejection of $\mathrm{M}$ electrons by $\mathrm{N}$ photons we divide the rate of ejection (transition rate to the M-electron continuum) by Nth power of the flux of photons

$$
Q^{(N, M)}=\frac{W_{e^{-}}(M)}{\left(F_{\text {photon }}\right)^{N}}
$$

Making use of the rate of transitions into the continuum from Fermi's Golden Rule applied to two-photon absorption in the dipole approximation (length gauge) and expressing the photon flux in terms of the electric field strength gives the generalized cross section for the ejection of two electrons by two photons as

$$
\begin{aligned}
\frac{d \sigma^{2 \omega}}{d E_{1} d \Omega_{1} d \Omega_{2}}= & \frac{2 \pi}{\hbar}(2 \pi \alpha)^{2} m^{2} \omega^{2} k_{1} k_{2} \\
& \times\left|\left\langle\Psi_{\mathbf{k}_{1} \mathbf{k}_{2}}^{-}\left|\sum \boldsymbol{\epsilon} \cdot \mathbf{r}_{i}\left[E_{i}+\hbar \omega-H_{0}\right]^{-1} \sum \boldsymbol{\epsilon} \cdot \mathbf{r}_{i}\right| \Phi_{i}\right\rangle\right|^{2}
\end{aligned}
$$

The units of this generalized cross section are, e.g., $\mathrm{cm}^{4} \mathrm{sec} / \mathrm{eV}$, and it is referred to as the triple differential cross section (TDCS) for two-photon double ionization. If we use a single pulse it can be calculated from the amplitudes $C\left(\mathbf{k}_{\mathbf{1}}, \mathbf{k}_{\mathbf{2}}\right)$ as follows.

The amplitudes for ionization extracted via Eq. (8) will generally depend on the parameters (intensity, bandwidth, etc.) of the radiation pulse that produced the wavepacket being analyzed. However, if the intensities are such that time-dependent perturbation theory (TDPT) gives an accurate description of the physical process and the pulse durations are not too short, then the amplitudes can be used to construct two-photon cross sections over the range of energies within the bandwidth of the pulse. That assertion would be rigorously true for single photon processes, because the firstorder TDPT transition amplitude can be factored into a dipole matrix element times a pulse dependent "shape function" depending on energy.

For two-photon processes an additional approximation is necessary to make that factorization, which we have verified to be essentially exact provided there are no intermediate state resonances that fall within the bandwidth of the pulse. We note that in the present context, the sequential threshold acts like an intermediate resonance. It is exact in the limit of long pulses. In this approximation, $\left|C\left(\mathbf{k}_{\mathbf{1}}, \mathbf{k}_{\mathbf{2}}\right)\right|^{2}$ is proportional to modulus squared of the matrix element in Eq. (10) and the proportionality constant depends on the parameters of the pulse. The lengthy derivation (Palacios et al. 2007, Palacios et al. 2008) of the working equations will not be reproduced here. The result is

$$
\frac{d \sigma^{2 \omega}}{d E_{1} d \Omega_{1} d \Omega_{2}}=\frac{8 \pi^{3}\left(\Delta E_{f i} / 2\right)^{2} m^{2} \hbar k_{1} k_{2}}{c^{2}\left|E_{0}\right|^{4}} \frac{\left|C\left(\mathbf{k}_{\mathbf{1}}, \mathbf{k}_{\mathbf{2}}\right)\right|^{2}}{\left|\widetilde{\mathfrak{F}}\left(E_{f}, E_{i}, \omega, T\right)\right|^{2}},
$$

and the effective energy shape function for the sine-squared radiation pulse is given by

$\widetilde{\mathfrak{F}}\left(E_{f}, E_{i}, \omega, T\right)=$ 


$$
\frac{6 e^{-i T\left(2 \omega-\Delta E_{f i}\right)}\left(-1+e^{i T\left(2 \omega-\Delta E_{f i}\right)}\right) \pi^{4}}{\left(2 \omega-\Delta E_{f i}\right)\left[T^{4}\left(2 \omega-\Delta E_{f i}\right)^{4}-20 \pi^{2} T^{2}\left(2 \omega-\Delta E_{f i}\right)^{2}+64 \pi^{4}\right]},
$$

where $E_{i}$ is the energy of the initial state, $E_{f}$ is the energy of the final state $\left(k_{1}^{2} / 2+k_{2}^{2} / 2\right.$ for double ionization), and $\Delta E_{f i}=E_{f}-E_{i}$.

It must be noted that above the threshold for sequential absorption the matrix element in Eq. (10) becomes singular, due to contributions from free-free overlaps between intermediate and final continuum states, as discussed by Horner et al. (Horner, McCurdy \& Rescigno 2008). So above that threshold the definition of the cross section fails, and we can only speak of the ionization probability distribution $\left|C\left(\mathbf{k}_{\mathbf{1}}, \mathbf{k}_{\mathbf{2}}\right)\right|^{2}$ for a particular pulse. Nonetheless, for a finite length pulse the quantity on the right hand side of Eq. (11) is still defined, and it is sometimes useful to plot it when comparing the double ionization probabilities that come from pulses of different intensities or durations - even though it is not in any sense a cross section. Obviously, in the case that there is more than one pulse the definition of the cross section in Eq. (9) fails and we must again focus on the pulse-dependent ionization probability distribution.

\subsection{Numerical Implementation}

In the calculations presented here, we represent the wave-function in terms of products of two-electron radial functions and coupled spherical harmonics, $\mathcal{Y}_{l_{1}, l_{2}}^{L, M}$,

$$
\Psi\left(\mathbf{r}_{1}, \mathbf{r}_{2}, t\right)=\sum_{l_{1}, l_{2}, L}^{l_{\max }, L_{\max }} \psi_{l_{1}, l_{2}, L}\left(r_{1}, r_{2}, t\right) \mathcal{Y}_{l_{1}, l_{2}}^{L, M=0}\left(\hat{\mathbf{r}}_{1}, \hat{\mathbf{r}}_{2}\right)
$$

including all $\left(L, l_{1}, l_{2}\right)$ configurations that can be constructed using some given value of $l_{\max }$ for the individual electron angular momenta and $L_{\max }$ for the total orbital angular momentum. Our calculations here are restricted to weak fields and an initial state with $L=0$, and convergence is achieved by including only $L=0,1,2$, as we have verified by carrying out calculations at the field intensities used here with $L_{\max }=3$. The radial degrees of freedom are discretized using a finite-element, discrete variable representation (FEM-DVR) with a product basis of Lobatto shape-functions (Rescigno \& McCurdy 2000, McCurdy et al. 2004).

The time-propagation in the presence of the field is carried out by using a CrankNicholson propagator (see details in (Palacios et al. 2007)) on the real part of the FEM-DVR grid. The end of the real part of the grid, $R_{0}$, must be chosen large enough to contain the spreading wavepacket over the duration of the pulse and avoid unphysical reflections from the grid boundaries. Once the pulse(s) is over, the resulting wavepacket is taken as the driving term for the scattered wave equation, which is solved with a complex portion extending beyond $R_{0}$ by 50 or 60 Bohr.

When there is more than one pulse or the pulse duration is long, larger grids and higher angular momenta must be used to converge the calculations. In the pump/probe calculations described below we used individual angular momenta up to $l=14$, and radial grids extending to $170 \mathrm{bohr}$. These are nonetheless much smaller grids than would have been necessary to calculate the amplitudes by other means not involving the solution of the scattered wave equation, Eq. (7). We emphasize again that given the parameters of a particular pulse, or sequence of pulses, the time propagation is carried out only once, and then the scattered wave equation can be solved for any energy within the pulse bandwidth. 


\section{One-color two-photon double ionization}

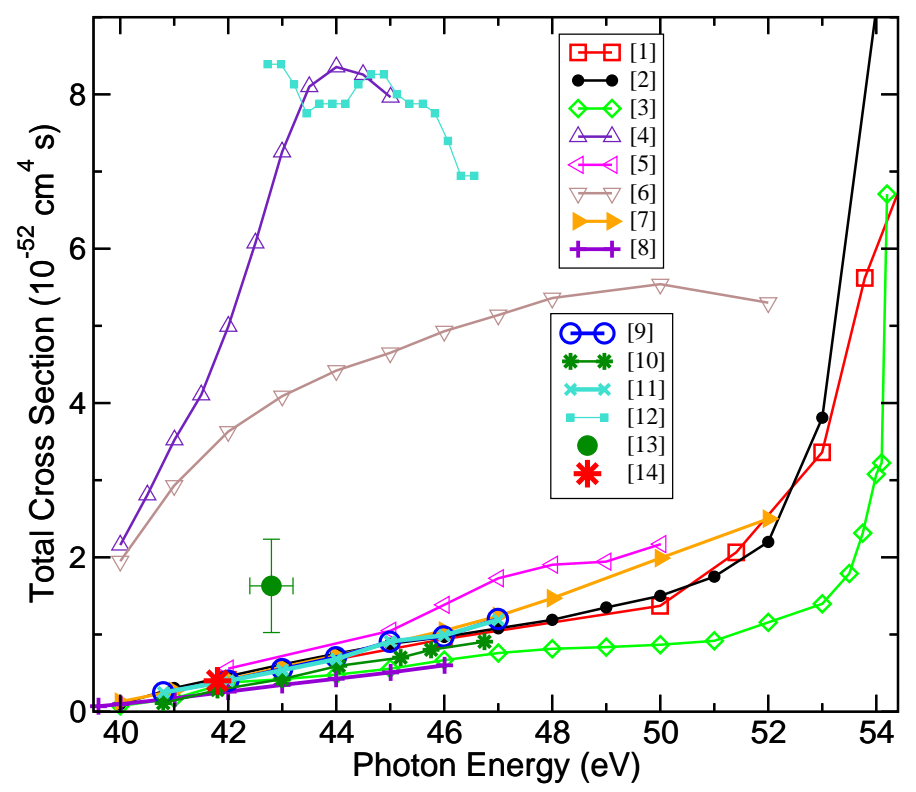

Figure 1. (Color online) Total two-photon double ionization cross sections as a function of photon energy. [1] 2fs results (Palacios et al. 2009a); [2] (Feist et al. 2008); [3] (Horner et al. 2007); [4] (Nikolopoulos \& Lambropoulos 2007); [5] (Ivanov \& Kheifets 2007); [6] (FC) (Foumouo et al. 2006); [7] (NC) (Foumouo et al. 2006); [8] (Hu et al. 2005); [9] (Piraux et al. 2003); [10] (Feng \& van der Hart 2003); [11] (Laulan \& Bachau 2003); [12] (Nikolopoulos \& Lambropoulos 2001); [13] (Sorokin et al. 2007); [14] (Hasegawa et al. 2005).

A number of investigators have investigated the problem of calculating the twophoton double ionization of helium in the nonsequential region, i.e., from $39.505 \mathrm{eV}$, which is the two-photon double ionization potential, to $54.4 \mathrm{eV}$, above which double ionization may proceed through a sequential absorption of each photon. Fig. 1 shows the total cross sections in this region, including our recent calculations (reference [1] in the figure) as well as previous work. With a few notable exceptions, there is agreement among the various theoretical calculations on the shape and magnitude of the cross section below $50 \mathrm{eV}$. The calculations also agree with one of the only two experimental data points available in the literature (references [13] and [14] in the figure). The rapid rise in the cross section above $52 \mathrm{eV}$, first predicted in a time-independent study (reference [3] in the figure) and subsequently verified in more recent time-dependent studies (labeled [1] and [2]), is caused by the virtual contribution of the singly ionized intermediate states associated with the energetically closed sequential process.

In Fig. 2 we show results obtained with pulses of different durations. The shorter the pulse, the fewer the number of time-dependent propagated wave packets that are required to cover the whole interval of photon energies. For instance, for a pulse duration of 450 as, the entire range plotted was computed by propagating only two pulses and extracting the cross sections within their bandwidths using Eq. (11). We find essentially perfect agreement with (Feist et al. 2008) when 3 fs pulses are used. 


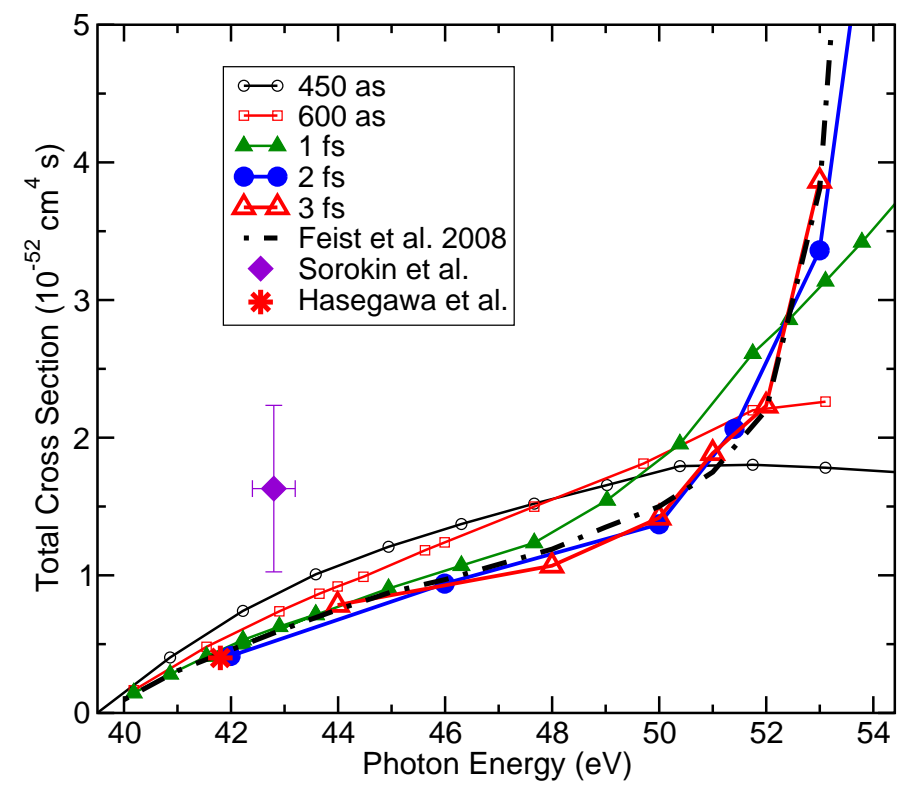

Figure 2. (Color online) Total two-photon double ionization cross sections as a function of photon energy. The current results are labeled with the corresponding pulse durations.

We can use the same procedure above the sequential ionization threshold to extract apparent cross sections via Eq. (11), with the understanding that they will increase with pulse duration and formally diverge in the CW limit (Palacios et al. 2009a). However, the relevant features in the region where sequential and nonsequential double ionization compete (above $54.4 \mathrm{eV}$ ) appear in the electron energy distributions. Fig. 3 shows double ionization probabilities as a function of the energy sharing of the ejected electrons (squared amplitudes defined in Eq. (8)). Pulse duration and intensity are fixed at $2 \mathrm{fs}$ and $10^{12} \mathrm{~W} \mathrm{~cm}{ }^{-2}$ respectively, and we plot different photon energies in a range from $45.5 \mathrm{eV}$ (22 cycles, where only nonsequential ionization is possible) to $70.3 \mathrm{eV}$ ( 34 cycles, already above the second sequential threshold, i.e., sequential ionization where the first photon produces $\mathrm{He}^{+}(2 \mathrm{~s}$ or $2 \mathrm{p})$ ). In order to make them comparable, probabilities have been extracted at the central frequency of each pulse.

In the nonsequential region, electron distributions are flat since the total energy available is equally shared between both ejected electrons. As we approach the first sequential threshold (see results for 26 and 28 cycles), the distribution shows two symmetric peaks, one at $E_{1}=E_{i}-\epsilon_{1 s}+\hbar \omega$ and the other $E_{1}=\epsilon_{1 s}+\hbar \omega$, where the orbital energy of the $\mathrm{He}^{+}$ion, $\epsilon_{1 s}$, and the energy of the ground state of $\mathrm{He}, E_{i}$, are negative and referenced to the zero of energy of the three separated particles. The heights of those peaks are controlled by the pulse duration and the product of the single ionization cross sections $\sigma^{H e}\left(E_{i}-\epsilon_{1 s}+\hbar \omega\right) \sigma^{H e^{+}}\left(\epsilon_{1 s}+\hbar \omega\right)$ (Palacios et al. 2009a). As the photon energy is further increased, we reach the second threshold for sequential ionization, in which the first step involves excitation ionization of the helium atom leaving the helium ion in its $2 \mathrm{~s}$ or $2 \mathrm{p}$ state at $65.4 \mathrm{eV}$. At energies above this point, 


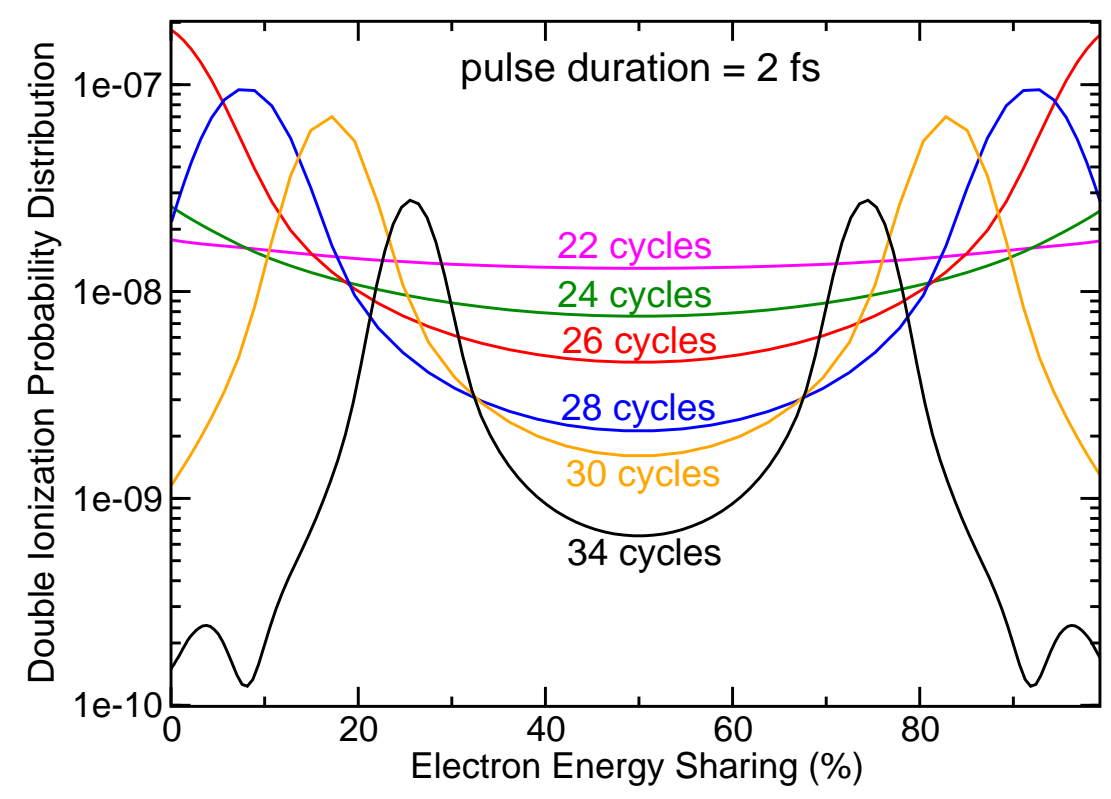

Figure 3. (Color online) Electron energy distributions for a fixed pulse duration of $2 \mathrm{fs}$ and intensity of $10^{12} \mathrm{~W} \mathrm{~cm}-2$ at different photon energies. Plotted energies go from below the first sequential threshold to above the second sequential threshold: 22 cycles $(45.5 \mathrm{eV}), 24$ cycles $(49.63 \mathrm{eV}), 26$ cycles $(53.8 \mathrm{eV}), 28$ cycles $(57.9 \mathrm{eV}), 30$ cycles $(62.05 \mathrm{eV})$ and 34 cycles $(70.3 \mathrm{eV})$.

we expect a second pair of peaks in the electron energy distributions placed at the energies given by $E_{1}=E_{i}-\epsilon_{2 p}+\hbar \omega$, and $E_{1}=\epsilon_{2 p}+\hbar \omega$, since the $2 \mathrm{p}$ and $2 \mathrm{~s}$ states of $\mathrm{He}^{+}$are degenerate. Those peaks appear in Fig. 3 for 34 cycles (corresponding to a photon energy of $70.3 \mathrm{eV}$ ) with a smaller intensity than the peaks associated with the first sequential threshold since the cross section for excitation ionization is smaller than that for simple ionization of He.

The positions of the second pair of sequential peaks are slightly shifted from their expected energies. For $70 \mathrm{eV}$ photons, the second pair of sequential peaks should appear at 4.59 and $56.4 \mathrm{eV}$ respectively, but they appear shifted to higher and lower energies, respectively, by nearly $2 \mathrm{eV}$. This behavior is due to the use of finite pulse lengths that allow the contributions of the two sequential processes proceeding through the $n=1$ and $n=2$ states of $\mathrm{He}^{+}$to interfere (Palacios et al. 2009a, Feist et al. 2009a). In fact, for infinite pulse durations, the sequential peaks would appear at the energies required by energy conservation. This shift and its pulse length dependence is better shown in Fig. 4, which shows the behavior of the corresponding electron energy distributions with varying pulse duration. The expected values for the ejected electrons through the first and second sequential threshold are plotted as vertical lines. In this figure we plot the apparent double ionization probability distributions obtained through Eq. (11). Their corresponding units are $10^{-52} \mathrm{~cm}^{4} \mathrm{~s}$ $\mathrm{eV}^{-1}$. For $2 \mathrm{fs}$ pulses we find the four distinct peaks mentioned above. As the pulse length is decreased these peaks broaden and even disappear at 250 as.

However tempting it may be to interpret the behavior shown in Fig. 4 as the extinguishing of the sequential process in favor of the nonsequential process for shorter 


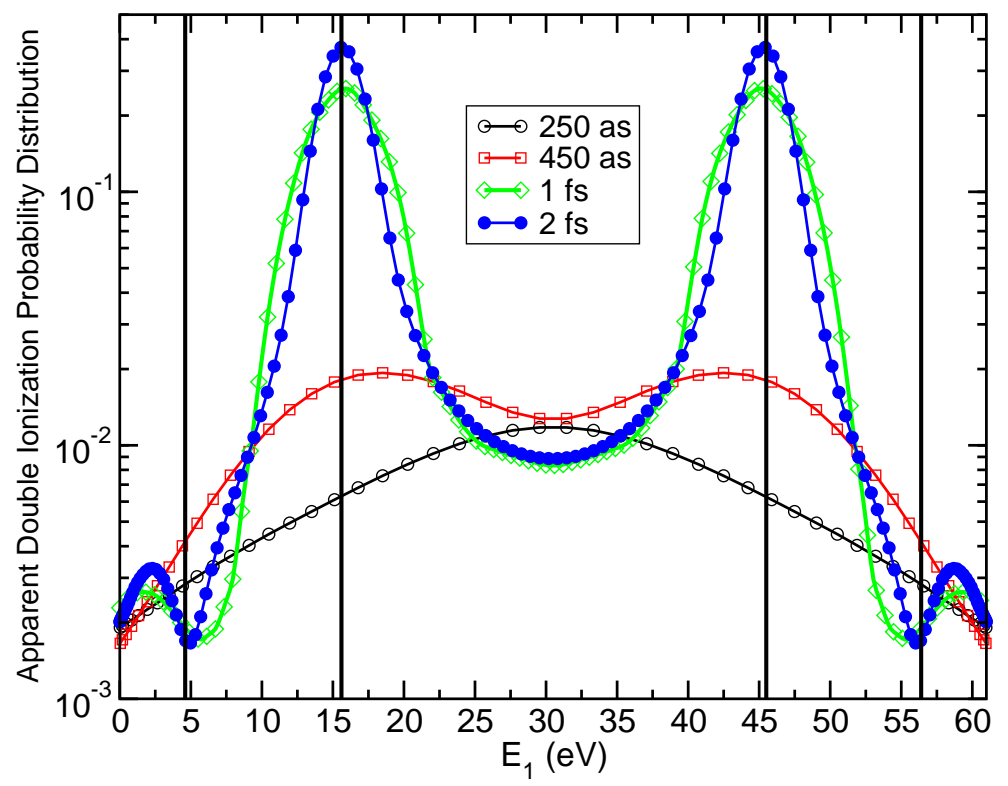

Figure 4. (Color online) Calculated electron energy distributions for pulse duration of $250 \mathrm{as}, 450 \mathrm{as}, 1 \mathrm{fs}$ and $2 \mathrm{fs}$ at $70 \mathrm{eV}$ and $\mathrm{I}=10^{12} \mathrm{~W} \mathrm{~cm}^{-2}$, showing the peaks due to ionization via the $n=2$ states of $\mathrm{He}^{+}$that shift with pulse duration.

pulses, the dependence of the electron energy distribution with pulse duration reflects nothing more than the Fourier broadening of the pulses. Indeed, we have shown that this behaviour is simply reproduced in time-dependent perturbation theory with a simple model that neglects electron correlation and screening entirely (see appendix in (Palacios et al. 2009a)). Therefore, to further explore the role of correlation in electron dynamics in the subfemtosecond regime we must analyze the electron angular distributions.

The qualitative behaviour of the angular distributions of the ejected electrons for femtosecond pulses is shown in Fig. 5. We plot the angular distributions obtained with a pulse of $1 \mathrm{fs}$ for both extreme situations: at a photon energy of $46 \mathrm{eV}$ and $50 \%$ energy sharing (below the sequential threshold and the total energy available equally shared) and $58 \mathrm{eV}$ and $10 \%$ energy sharing (above the threshold and right on top of one of the sequential peaks for the energy distribution). When both electrons are ejected simultaneously by a nonsequential absorption process $(46 \mathrm{eV})$, the angular distributions show a propensity for back-to-back ejection. This is an expected behaviour due to the important role of correlation between the electrons in this process. On the other hand, for $58 \mathrm{eV}$ at $10 \%$ energy sharing, the angular distributions are fairly well described by a product of dipole patterns $\left(\cos ^{2}\left(\theta_{1}\right) \cos ^{2}\left(\theta_{2}\right)\right)$. Here we see a largely uncorrelated angular behavior, with each electron independently ejected by each photon.

A different electron dynamics arises in the sequential region when using subfemtosecond pulses. In Fig. 6, we plot, together with the one fs results, the electron angular distributions for 450 and 250 as pulses for a photon energy of $58 \mathrm{eV}$ and $10 \%$ energy sharing. We show that shortening the pulse lengths in the subfemtosecond 


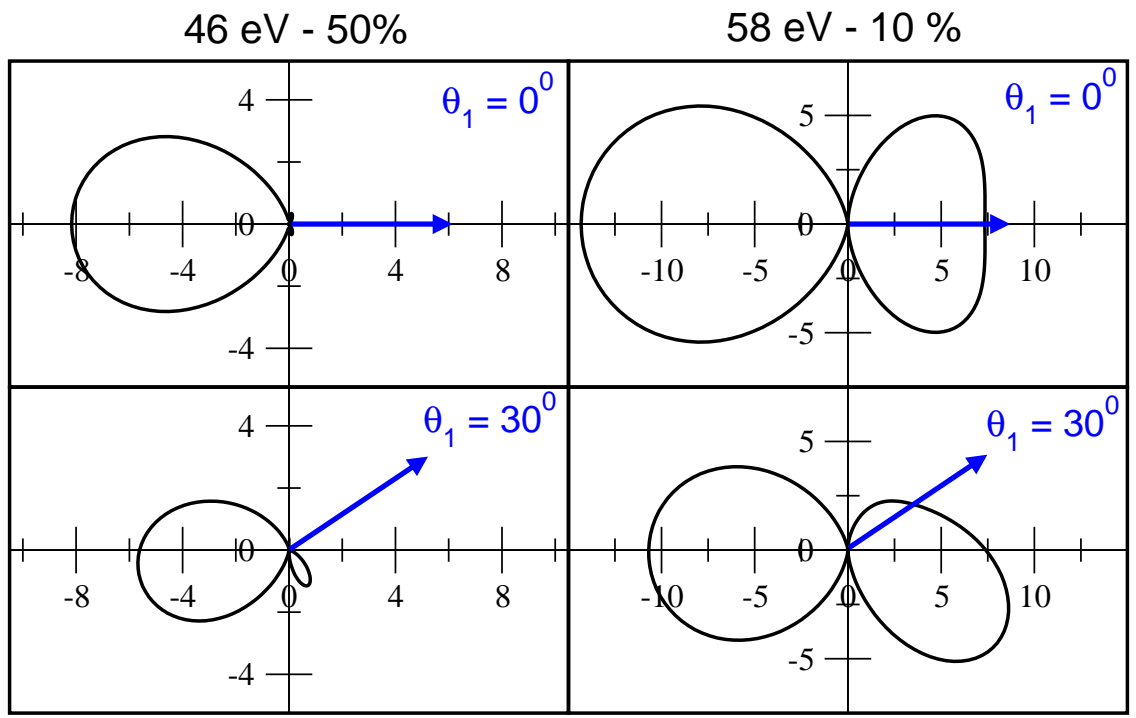

Figure 5. (Color online) Angular distributions at $46 \mathrm{eV}$ photon energy with 50\% energy sharing (left column) and $58 \mathrm{eV}$ with $10 \%$ energy sharing(right column). Each row corresponds to a different angle of one electron fixed with respect to the polarization vector (horizontal axis). Results are shown for a pulse duration of one fs and calculated with $l_{\max }=7$.

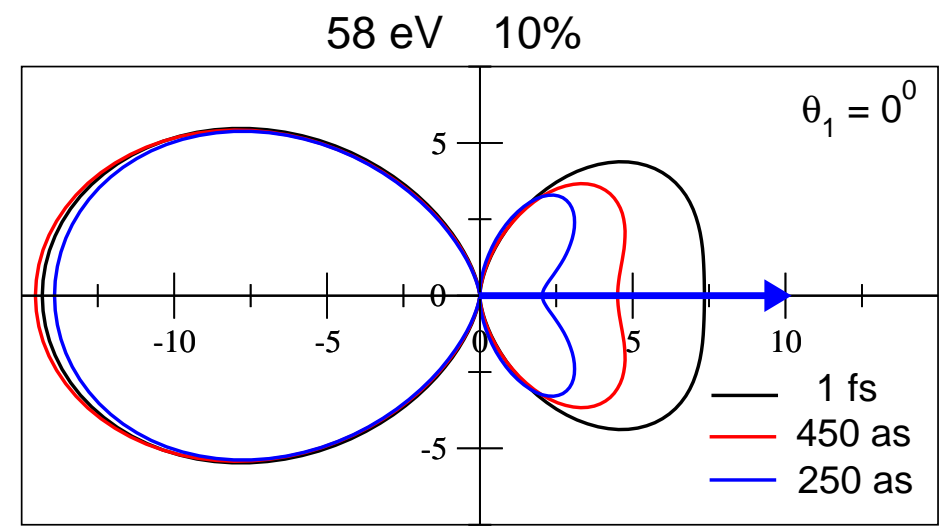

Figure 6. (Color online) Angular distributions from calculations including up to $l_{\max }=7$ for a photon energy of $58 \mathrm{eV}$ with energy sharing of $10 \%$ and pulse lengths of $1 \mathrm{fs}, 450$ as and 250 as. Note that the all cross sections are normalized to the $1 \mathrm{fs}$ TDCS for easier comparison of the shapes.

regime leads to an increasingly back-to-back ejection pattern, i.e., we are probing a different dynamics as the pulse length is shortened: at those energy sharings where femtosecond pulses show dipole patterns related to uncorrelated processes, subfemtosecond pulses reveal the signatures of the nonsequential mechanism of ejection as also found by (Feist et al. 2009b). Therefore, while the pulse length dependence of the electron energy distributions can be simply explained by the Fourier broadening 
of the subfemtosecond pulses, the variation of the angular distributions with pulse duration is actually probing the electron dynamics of photoejection, disentangling the features of sequential and nonsequential ionization processes (Palacios et al. 2009a).

Since the angular distributions produced in two-photon sequential and nonsequential ionization are strikingly different, they leave a clear signature in the nuclear recoil distributions. The ion recoil momentum $\mathbf{Q}$ accompanying double ionization is simply $-\mathbf{k}_{1}-\mathbf{k}_{2}$. We can therefore define a nuclear recoil cross section, at a particular energy sharing $E_{1}$, in terms of the two-photon TDCS as

$$
\frac{d \sigma}{d^{3} \mathbf{Q} d E_{1}}=\int d \Omega_{1} \int d \Omega_{2} \frac{d \sigma^{2 \omega}}{d E_{1} d \Omega_{1} d \Omega_{2}} \delta^{3}\left(\mathbf{Q}+\mathbf{k}_{1}+\mathbf{k}_{2}\right) .
$$

The distribution of ion recoil momentum, integrated over all energy sharings, presents an attractive target for experimental detection, since it does not require detection of the photoemitted electrons. A procedure for carrying out the numerical evaluation of the integral in Eq. (14) is outlined in (Horner, McCurdy \& Rescigno 2008).

Fig. 7 shows ion momentum recoil distributions for a photon energy of $52 \mathrm{eV}$, which is close to the sequential ionization threshold. (The raggedness in the figures is not physical, but rather the result of numerical error in the plotting renditions). These distributions were obtained from calculations using a 2 fs pulse. At $52 \mathrm{eV}$, the electron energy sharing distributions are peaked at extreme unequal energy sharing, since "virtual sequential" ionization is beginning to dominate the electron dynamics (Horner, Rescigno \& McCurdy 2008). However, inspection of the TDCS at this photon energy shows that the electron dynamics is still dominated by back-toback ejection along the direction of photon polarization (Horner et al. 2010), reflecting the importance of correlation in the nonsequential region. The resulting ion recoil distributions show the development of prominent rings at unequal energy sharing and these rings dominate the ion recoil distribution, even when integrated over all energy sharings. In purely sequential ionization, the faster electron can give a strong kick to

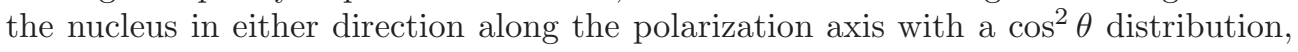
while the slower electron gives a smaller kick that either adds to or subtracts from the first. The result would be a four-ringed pattern in the nuclear recoil distribution. However just below the sequential threshold, as Fig. 7 shows, the outer rings at unequal energy sharing are strongly suppressed, since back-to-back ejection is dominant. 
Two-photon double ionization of He
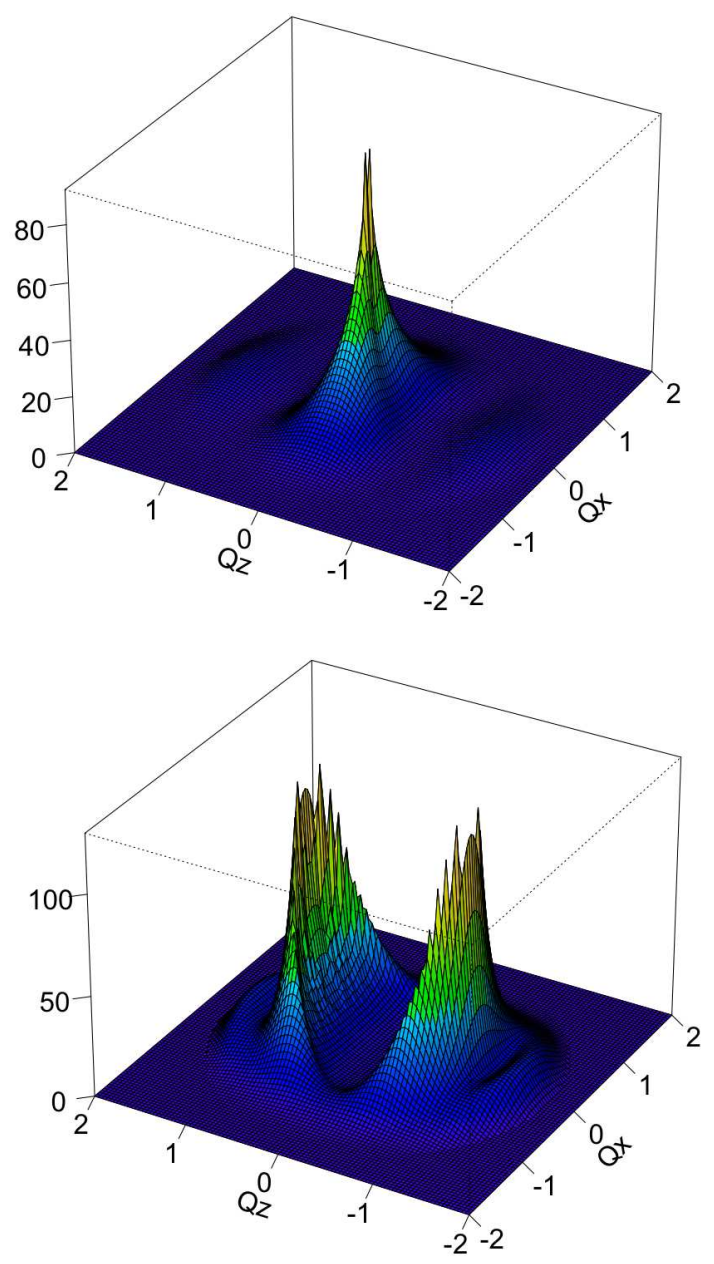
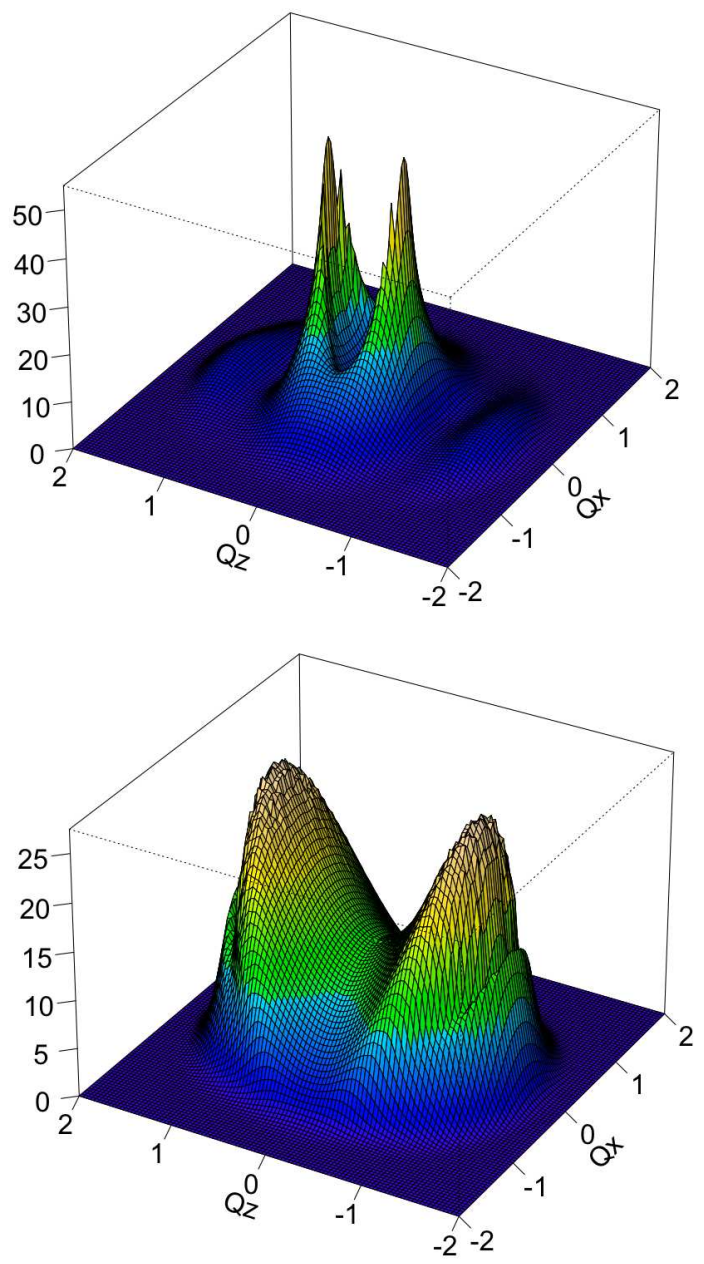

Figure 7. (Color online.) Nuclear recoil momentum distributions, integrated over $\mathrm{Q}_{y}$, at $52 \mathrm{eV}$ photon energy for calculations using a $2 \mathrm{fs}$ pulse. Polarization is along the z-axis. Energy sharings, left to right: top row, 50\%, 70\%; bottom row, $90 \%$, integrated over energy sharing. 


\section{Two-color time-delayed two-photon double ionization}

Pump/probe experiments with subfemtosecond pulses of different colors that are both in the UV or XUV provide a way to interrogate the electron dynamics of correlated states, for example doubly excited autoionizing states. The large bandwidths of subfemtosecond pulses are both an essential advantage and a complication in those experiments, since the pump pulse can create a wave packet of many excited states of the target, and the probe, especially if it is at ionizing energies, can interrogate all of them simultaneously. Such experiments on two-electron atoms have been modeled in detailed theoretical calculations ( $\mathrm{Hu} \&$ Collins 2006, Morishita et al. 2007, Palacios et al. 2009b) that suggest they have the power to expose correlation effects not otherwise accessible experimentally, but such UV/XUV pump/probe experiments are only beginning to be realized.

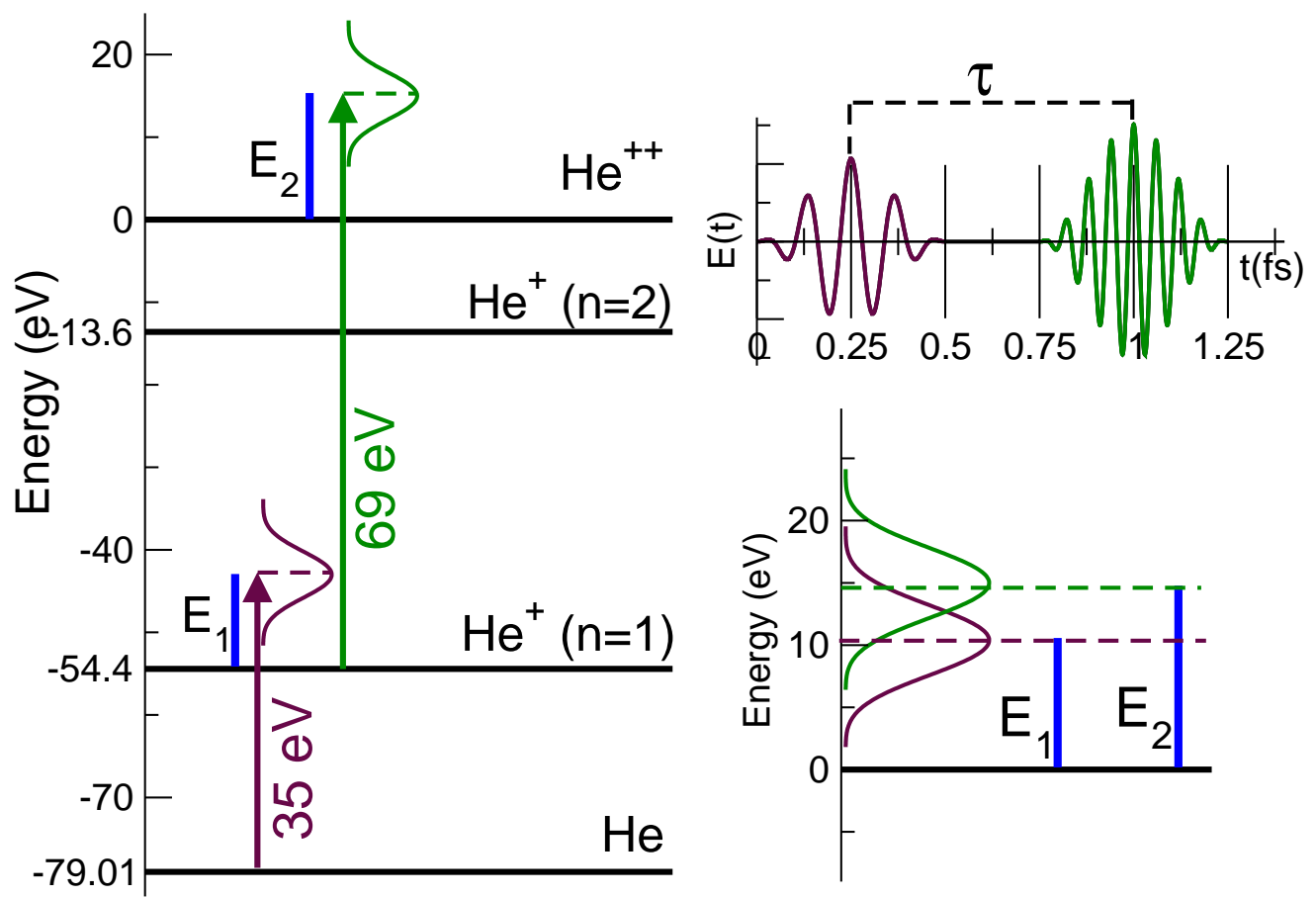

Figure 8. (Color online.) Left: Schematic of the energetics for a two-color twophoton double ionization process in helium. Top Right: Electromagnetic field as function of time showing two pulses of 500 as, with 35 and $69 \mathrm{eV}$ respectively and a time delay of $\tau=750$ as between them. Bottom right: Expected energies for electrons ejected from each sequential process.

Here we discuss an aspect of such measurements that is connected specifically to the bandwidths of the pulses, namely the case where the atom is doubly ionized mainly through a sequential process that ejects electrons with similar energies. For this purpose, we choose two pulses of central frequencies of 35 and $69 \mathrm{eV}$, ejecting electrons with energies centered around $10.4 \mathrm{eV}$ and $14.6 \mathrm{eV}$ respectively. A schematic representation of the energetics involved in the process is shown in Fig. 8. In the sequential process, the $35 \mathrm{eV}$ photon is absorbed first leaving the helium ion in its 
ground state, and the $69 \mathrm{eV}$ photon ionizes the ion, ejecting the second electron. Since we are using subfemtosecond pulses with large energy bandwidths, those electron energy distributions will overlap, and because electrons are indistinguishable, an electron ejected with a given energy within the overlapping bandwidths can have been ejected by either pulse. Therefore, when the pulses are absorbed with a time delay between them, the resulting photoelectron energies probabilities show interference oscillations that contain information about the sequential ejection process revealed by variations with both the time delay and the pulse durations.
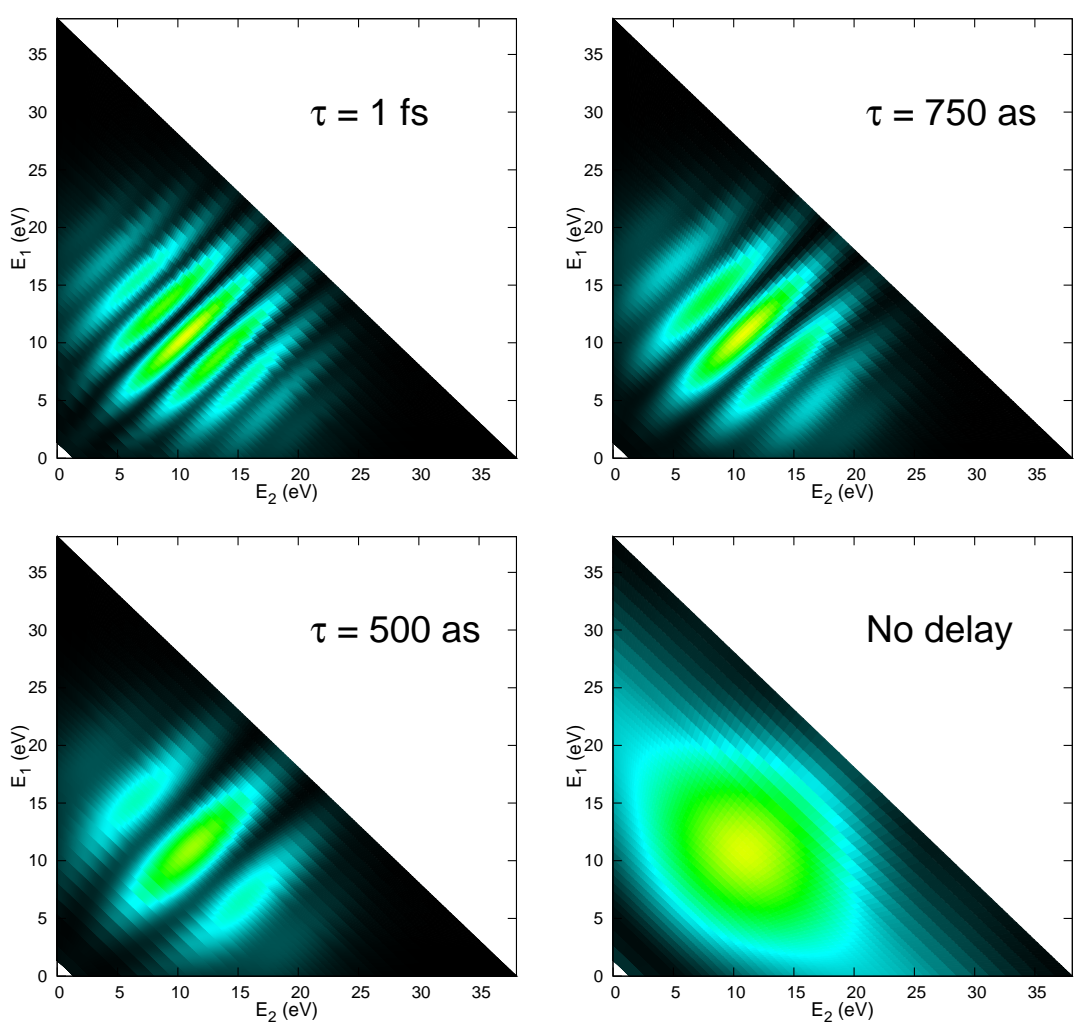

Figure 9. (Color online.) Joint electron energy distributions for pulse durations $T_{1}=T_{2}=500$ as. Each panel corresponds to a different time delay between pulses.

Quantum interference arising from exchange symmetry was predicted some years ago (Végh \& Macek 1994) and observed in coincidence measurements of photoelectrons and Auger electrons (Schwarzkopf \& Schmidt 1996, Viefhaus et al. 1998). For that interference phenomenon to be observed, the photoelectron energy must lie within the Auger width of the energy of the Auger electron, and the only adjustable condition in the experiment is the photoelectron energy. Here the variable time-delay, energies and bandwidths of two subfemtosecond UV pulses open the possibility of a generally applicable experimental technique that is sensitive to the exchange symmetry, and therefore the spin coupling of the ejected electrons.

In order to explore the time delay dependence of these two-electron interference 
patterns, we carried out calculations solving the time-dependent Schrödinger equation for different time delays, with fixed choices of the pulse durations. We choose an intensity of $10^{12} \mathrm{~W} \mathrm{~cm}^{-2}$ for the first pulse centered at $35 \mathrm{eV}$ and $2 \times 10^{12} \mathrm{~W} \mathrm{~cm}^{-2}$ for the second one at $69 \mathrm{eV}$ in all cases. For the time-propagation, as well as for the extraction of the corresponding amplitudes, we use the methodology described in Section 2 with the electromagnetic field given by

$$
\mathbf{E}(t)=\left(E^{\omega_{1}}(t)+E^{\omega_{2}}(t)\right) \boldsymbol{\epsilon},
$$

corresponding to two pulses with the same polarization vector $\epsilon$ but different colors. On the time intervals where each individual pulse is nonzero, the terms in Eq. (15) are specified by

$$
\begin{array}{lll}
E^{\omega_{1}}(t)=E_{0}^{(1)} f^{(1)}(t) \sin \left(\omega_{1} t\right), & t \in\left[0, T_{1}\right] \\
E^{\omega_{2}}(t)=E_{0}^{(2)} f^{(2)}\left(t-t_{2}\right) \sin \left(\omega_{2}\left(t-t_{2}\right)\right), & t \in\left[t_{2}, T\right]
\end{array}
$$

where $f^{(i)}(t)$ is chosen to be a sine-squared pulse envelope. The pulse durations are $T_{1}$ and $T_{2} ; \tau$ is the time delay between the centers of the pulses as sketched in the top right panel of Fig. 8 , and $t_{2}=\tau+\left(T_{1}-T_{2}\right) / 2$.

In Fig. 9, electron energy-sharing distributions, $k_{1} k_{2} \int d \Omega_{1} \int d \Omega_{2}\left|C\left(\mathbf{k}_{1}, \mathbf{k}_{2}\right)\right|^{2}$, as functions of the energy of each electron are plotted for the case that both pulses are 500 as long, and show the entire energy-sharing patterns for different time delays that would be obtained in a coincident detection of the electron energies integrated over the angles of photoejection. To produce each panel of Fig. 9 double ionization probabilities have been extracted from a single wave packet solution of the TDSE for a range of total energies centered at the total electron energy that results from the sum of the central energies of the pulses $(104 \mathrm{eV})$ less the total binding energy of the helium atom. The bandwidths seen in these plots are determined by the pulse durations, i.e., longer pulses would lead to a narrower spread in the total available energy to be shared by the electrons.

When both pulses reach the target simultaneously $(\tau=0)$ there is a single peak with maximum ionization probability centered at equal energy sharing at the total energy determined by the central frequencies of the pulses. As the time delay increases (to 500 as, 750 as and 1 fs in Fig. 9), an increasing number of oscillations appear in these joint energy distributions. The separations of the peaks as function of the difference in the electron energies, $E_{2}-E_{1}$, is a measure of the time delay between the pulses, and it can be estimated from this figure for pulses of equal duration to be approximately $2 \pi \hbar / \tau$.

We can understand the origin of these interferences in atoms by using a simple model from second-order time-dependent perturbation theory detailed in (Palacios et al. $2009 b$ ) that completely neglects electron-electron interaction in the doubly ionized state, as well as electron correlation and screening in the sum over intermediate states. Applying this model to the cases in which the pulses do not overlap in time, an approximate amplitude for double ionization in the range of energies of interest here can be written as

$$
\begin{aligned}
C\left(\mathbf{k}_{1}, \mathbf{k}_{2}\right) \approx\left(\frac{-i}{\hbar}\right)^{2} & \frac{1}{\sqrt{2}} \\
& \times\left\{e^{i\left(\alpha\left(k_{1}\right)+\omega_{2}\right) \Delta t} J_{2}\left(\alpha\left(k_{1}\right)\right) J_{1}\left(\beta\left(k_{1}\right)\right)\left\langle\varphi_{\mathbf{k}_{2}}^{(-)}|\mu| \varphi_{1 s}^{H e^{+}}\right\rangle\left\langle\psi_{\mathbf{k}_{1}, 1 s}^{-}|\mu| \Phi_{0}\right\rangle\right. \\
& \left.+e^{i\left(\alpha\left(k_{2}\right)+\omega_{2}\right) \Delta t} J_{2}\left(\alpha\left(k_{2}\right)\right) J_{1}\left(\beta\left(k_{2}\right)\right)\left\langle\varphi_{\mathbf{k}_{1}}^{(-)}|\mu| \varphi_{1 s}^{H e^{+}}\right\rangle\left\langle\psi_{\mathbf{k}_{2}, 1 s}^{-}|\mu| \Phi_{0}\right\rangle\right\}
\end{aligned}
$$


In Eq. (17) the amplitudes for the two sequential ionization events appear separately. The first is the dipole matrix element, $\left\langle\psi_{\mathbf{k}, 1 s}^{-}|\mu| \Phi_{0}\right\rangle$ between the ground state of the helium atom, $\Phi_{0}$, and the continuum state, $\psi_{\mathbf{k}, 1 s}^{-}$, for the singly ionized intermediate state with an electron scattering from the ground state of $\mathrm{He}^{+}$. The second ionization amplitude, $\left\langle\varphi_{\mathbf{k}}^{(-)}|\mu| \varphi_{1 s}^{H e^{+}}\right\rangle$, is the dipole matrix element for ionization of $\mathrm{He}^{+}$in which $\varphi_{\mathbf{k}}^{(-)}$denotes a momentum-normalized Coulomb scattering function with charge $Z=2$.

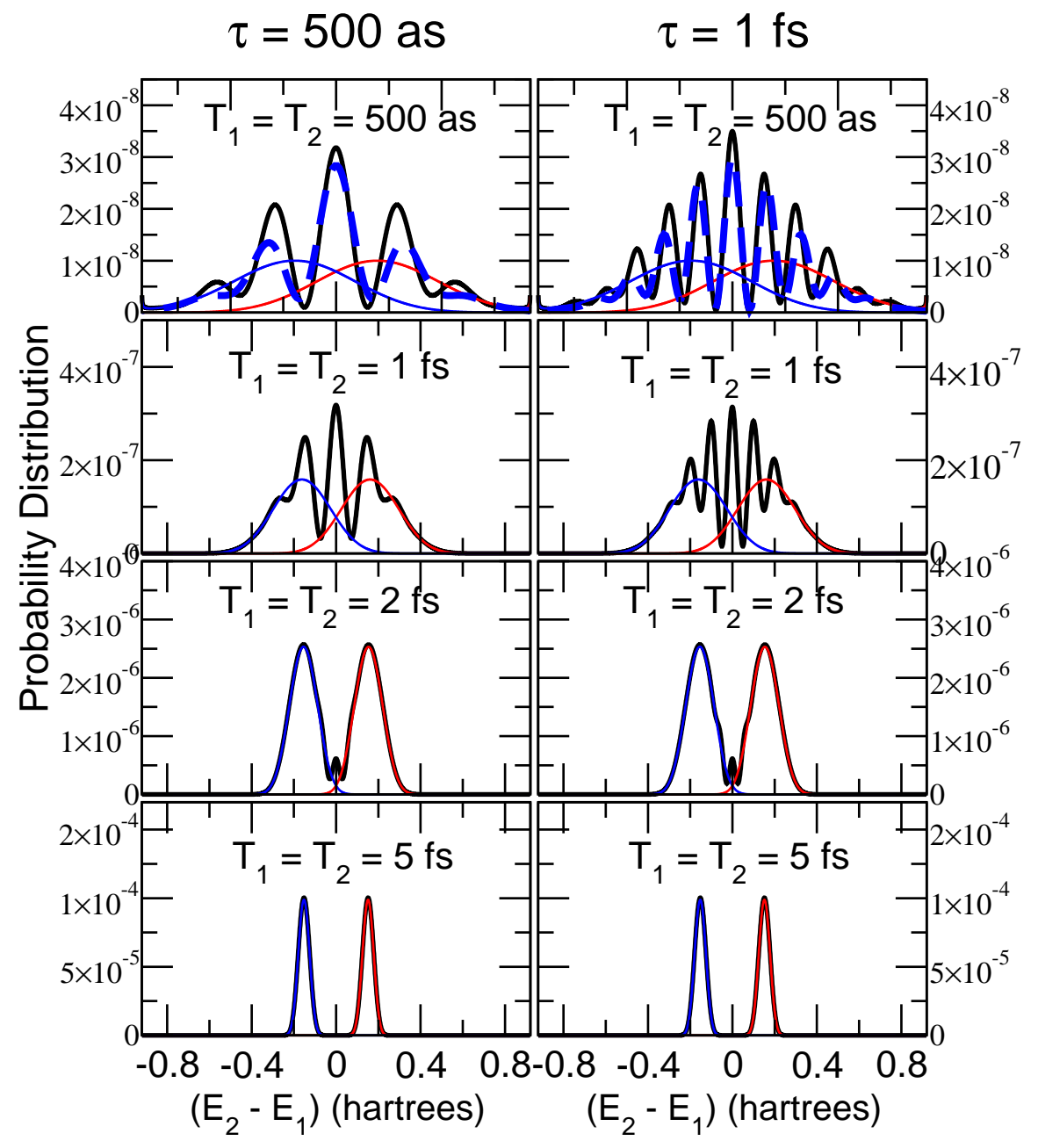

Figure 10. (Color online.) Electron energy joint distributions determined at the central energies of the pulses. Left column: delay of 500 as. Right column: delay of $1 \mathrm{fs}$. Each row corresponds to different pulse durations (in all cases both pulses have same pulse duration). Black full lines: results obtained with the model (see text). Full thin curves are the squared values of $J_{i}$ defined in Eq. (18) whose energy overlapping leads to the interference fringes. Dashed lines: ab initio calculations.

Products of these dipole matrix elements corresponding to the two possible orders of ejection (electron 1 before 2 and 2 before 1) appear added together in Eq. (17) with 
coefficients that arise from time-dependent perturbation theory. Those coefficients are functions of the three quantities $\alpha\left(k_{i}\right)=k_{1}^{2} / 2+k_{2}^{2} / 2-k_{i}^{2} / 2-E_{1 \mathrm{~s}\left(\mathrm{He}^{+}\right)}-\omega_{2}$, $\beta\left(k_{i}\right)=k_{i}^{2} / 2+E_{1 \mathrm{~s}\left(\mathrm{He}^{+}\right)}-E_{\mathrm{He}}-\omega_{1}$, and $\Delta t=\tau+\left(T_{1}-T_{2}\right) / 2$. These quantities appear as parameters in integrals that depend on the pulse shapes and have the form

$$
J_{i}(\gamma) \equiv E_{0}^{(i)} \int_{0}^{T_{i}} e^{i \gamma t} f^{(i)}(t) d t
$$

where $f^{(i)}(t)$ appears in Eq.(16) and here has the form $\sin ^{2}\left(\pi t / T_{i}\right)$. It is these functions, $J_{1}$ and $J_{2}$, that reflect the bandwidths of the two pulses. To simplify this model even further, the dipole amplitudes can be approximated in terms of the square roots of the single photoionization cross sections of $\mathrm{He}$ and $\mathrm{He}^{+}$, thereby neglecting their phases. Thus the model gives the double ionization probabilities in terms of quantities pertaining only to the two separate sequential steps.

The coherent addition of the two terms in Eq. (17), corresponding to the "direct" and "exchange" processes in which the electrons are emitted in different orders, is responsible for the oscillations in the double ionization probabilities, $\left|C\left(\mathbf{k}_{1}, \mathbf{k}_{2}\right)\right|^{2}$, as a function of the energies of the electrons. In Fig. 10, the probability distribution $k_{1} k_{2} \int d \Omega_{1} \int d \Omega_{2}\left|C\left(\mathbf{k}_{1}, \mathbf{k}_{2}\right)\right|^{2}$ obtained from the model is plotted together with the products of the $J_{i}$ integrals appearing in Eq. (17). When the pulse durations are short enough that the corresponding energy bandwidths lead to products $J_{2}\left(\alpha\left(k_{1}\right)\right) J_{1}\left(\beta\left(k_{1}\right)\right)$ and $J_{2}\left(\alpha\left(k_{2}\right)\right) J_{1}\left(\beta\left(k_{2}\right)\right)$ that overlap, the energy-sharing distributions show the interference pattern. However, as we increase the pulse duration to $5 \mathrm{fs}$, decreasing the energy bandwidth, there is no overlapping region and the oscillations disappear, leading to the two well defined peaks that we would expect from the sequential double ionization process. In Fig. 10 we have also plotted in the top panels the accurate results obtained in our ab initio calculations, showing that remarkably good quantitative agreement can be obtained with the model in spite of the extreme oversimplification of the physics it represents.

The simple model shows how the indistinguishability of the electrons, and the fact that their spins must remain coupled in the same way as in the initial state of the atom (singlet in this case) produces the interference pattern. Thus the spin coupling is of the outgoing electrons is being probed by the interference pattern. If the initial state of He were a triplet, the plus sign in Eq. (17) would be a minus, and minima would appear where there are maxima, and vice versa, in the interference patterns in Figs. 9 and 10.

However, we should remark that, although the sequential model correctly describes the features of the photoelectron energy-sharing distribution, it fails to describe the angular distributions of photoelectrons, especially for short time delays (Palacios et al. 2009b), where there exists a strong interaction in the final state. While the sequential model predicts a product of dipole patterns, $\cos ^{2}\left(\theta_{1}\right) \cos ^{2}\left(\theta_{2}\right)$, for the angular distributions of the ejected electrons, back-to-back ejection is observed for pulses with no time delay, which persists, in spite of an apparent tendency to the dipole pattern, up to around $\tau=1 \mathrm{fs}$.

\section{Conclusion}

Free-electron lasers, like FLASH, can produce ultrashort pulses of sufficient intensity that multiphoton, multiple ionization processes become the dominant processes when they collide with atoms and molecules. Such experiments are expected to be able to 
probe correlated electron dynamics in atoms and in complex molecules, and observe them in combination with vibration, dissociation and chemical reactions.

In spite of the apparent simplicity of the target, or perhaps because of it, the body of experiments and accurate theory on two-photon short pulse ionization of the helium atom will be the urtext for such processes in more complicated systems, and we can expect many further studies on this system. The majority of those experiments, and much of the theory, have yet to be done, and they are not motivated primarily by need to understand the correlated electron dynamics of three body Coulomb breakup, but rather by the question of how that dynamics is revealed in ultrafast experiments.

For example, pump/probe experiments on the doubly excited states of helium, long a subject of theoretical and experimental interest because of their highly correlated nature, will be the subject of these investigations now that free-electron lasers and other XUV sources are making them a practicality. Perhaps most importantly, the interplay between theory and experiment for this system, for which we can expect that essentially exact calculations can be performed, will allow the benchmarking of experiments in which sensitive detection schemes must be employed to disentangle the various channels of ionization processes possible under given conditions.

\section{Acknowledgments}

This work was performed under the auspices of the US Department of Energy by the University of California Lawrence Berkeley National Laboratory under Contract DEAC02-05CH11231 and was supported by the U.S. DOE Office of Basic Energy Sciences,

Division of Chemical Sciences. CWM acknowledges support from the National Science Foundation (Grant No. PHY-0604628).

\section{References}

Alt E O \& Mukhamedzhanov A M 1993 Phys. Rev. A 47, 2004.

Colgan J \& Pindzola M S 2002 Phys. Rev. Lett. 88, 173002.

Feist J, Nagele S, Pazourek R, Persson E, Schneider B I, Collins L A \& Burgdörfer J 2008 Phys. Rev. A 77, 043420 .

Feist J, Pazourek R, Nagele S, Persson E, Schneider B I, Collins L A \& Burgdorfer J 2009a J. Phys. B: At. Mol. Phys. 42, 134014.

Feist J, Nagele S, Pazourek R, Persson E, Schneider B I, Collins L A \& Burgdorfer J 2009 b Phys. Rev. Letts. 103, 063002.

Feng L \& van der Hart H W 2003 J. Phys. B: At. Mol. Opt. Phys. 36, L1.

Foumouo E, Antoine P, Piraux B, Malegat L, Bachau H \& Shakeshaft R 2008 J. Phys. B: At. Mol. Opt. Phys. 41, 1.

Foumouo E, Antoine P \& Piraux H B B 2008 New Journal of Physics 10, 025017.

Foumouo E, Kamta G L, Edah G \& Piraux B 2006 Phys. Rev. A 74, 063409.

Hasegawa H, Takahashi E J, Nabekawa Y, Ishikawa K L \& Midorikawa K 2005 Phys. Rev. A 71, 023407.

Horner D A, McCurdy C W \& Rescigno T N 2008 Phys. Rev. A 78, 043416.

Horner D A, McCurdy C W \& Rescigno T N 2010 Phys. Rev. A 81, xxxx.

Horner D A, Morales F, Rescigno T N, Martín F \& McCurdy C W 2007 Phys. Rev. A 76, 030701(R).

Horner D A, Rescigno T N \& McCurdy C W 2008 Phys. Rev. A 77, 030703(R).

Hu S X, Colgan J \& Collins L A 2005 J. Phys. B: At. Mol. Opt. Phys. 38, L35.

Hu S X \& Collins L A 2006 Phys. Rev. Lett. 96, 073004.

Ivanov I A \& Kheifets A S 2007 Phys. Rev. A 75, 033411.

Kadyrov A S, Mukhamedzhanov A M \& Stelbovics A T 2003 Phys. Rev. A 67, 024702.

Kheifets A S \& Ivanov I A 2006 J. Phys. B: At. Mol. Opt. Phys. 39, 1731.

Krausz F \& Ivanov M 2009 Rev. Mod. Phys. 81, 163-234. 
Kurka M, Rudenko A, Foucar L, Kuhnel K U, Jiang Y H, Ergler T, Havermeier T, Smolarski M, Schossler S, Cole K, Schoffler M, Dorner R, Gensch M, Dusterer S, Treusch R, Fritzsche S, Grum-Grzhimailo A N, Gryzlova E V, Kabachnik N M, Schroter C D, Moshammer R \& Ullrich J 2009 Journal of Physics B: At. Mol. Opt. Phys. 42, 141002.

Laulan S \& Bachau H 2003 Phys. Rev. A 68, 013409.

Lunnemann S, Kuleff A I \& Cederbaum L S 2008 J. Chem. Phys. 129, 104305.

McCurdy C W, Baertschy M \& Rescigno T N 2004 J. Phys. B: At. Mol. Opt. Phys. 37, R137.

Morishita T, Watanabe S \& Lin C D 2007 Phys. Rev. Lett. 98, 083003.

Mukhamedzhanov A M, Kadyrov A S \& Pirlepesov F 2006 Phys. Rev. A 73, 012713.

Nikolopoulos L A A \& Lambropoulos P 2001 J. Phys. B: At. Mol. Opt. Phys. 34, 545.

Nikolopoulos L A A \& Lambropoulos P 2007 J. Phys. B: At. Mol. Opt. Phys. 40, 1347.

Palacios A, McCurdy C W \& Rescigno T N 2007 Phys. Rev. A 76, 043420.

Palacios A, Rescigno T N \& McCurdy C W 2008 Phys. Rev. A 77, 032716.

Palacios A, Rescigno T N \& McCurdy C W 2009a Phys. Rev. A 79, 033402.

Palacios A, Rescigno T N \& McCurdy C W 2009 b Phys. Rev. Lett. 103, 253001.

Piraux B, Bauer J, Laulan S \& Bachau H 2003 Eur. Phys. J. D 26, 7.

Proulx D, Pont M \& Shakeshaft R 1994 Phys. Rev. A 49, 1208.

Remacle F \& Levine R D 2006 Proc. Nat. Academy of Sciences 103, 6793.

Rescigno T N \& McCurdy C W 2000 Phys. Rev. A 62, 032706.

Rudenko A, Foucar L, Kurka M, Ergler T, Kühnel K U, Jiang Y H, Voitkiv A, Najjari B, Kheifets A, Lüdemann S, Havermeier T, Smolarski M, Schössler S, Cole K, Schöffler M, Dörner R, Düsterer S, Li W, Keitel B, Treusch R, Gensch M, Schröter C D, Moshammer R \& Ullrich J 2008 Phys. Rev. Lett. 101, 073003.

Schwarzkopf O \& Schmidt V 1996 J. Phys. B: At. Mol. Opt. Phys. 29, 3023-3032.

Sekikawa T, Kosuge A, Kanai T \& Watanabe S 2004 Nature 432, 605.

Shakeshaft R 2007 Phys. Rev. A 76, 063405.

Sorokin A A, Weelhöfer M, Bobashev S V, Tiedtke K \& Richter M 2007 Phys. Rev. A 75, 051402(R). Ullrich J 2009 (private communication).

V. Ayvazyan, et al. 2006 Eur. Phys. J. D 37, 297.

Végh L \& Macek J H 1994 Phys. Rev. A 50, 4031-4035.

Viefhaus J, Snell G, Hentges R, Wiedenhöft M, Heiser F, Geßner O \& Becker U 1998 Phys. Rev. Lett. 80, 1618-1621. 